\title{
PERCEPÇÃO E CONSUMO AMBIENTAL: ANÁLISE DOS FUNCIONÁRIOS DE UMA EMPRESA DE SOFTWARE E SERVIÇOS
}

\author{
Marco Antonio Sartori \\ Graduação em Sistema de Informação. Especialista em Engenharia de Software. Mestrado em \\ Administração. Atualmente é colaborador da Wealth Systems. \\ E-mail: msartoridc@gmail.com
}

Emanuel Campigotto Sandri

Graduação em Administração. Mestrado em Administração. Doutorando em Admnistração. Atualmente é Professor da Pontifícia Universidade Católica do Paraná.

E-mail: emanuel.sandri@hotmail.com

Júlia Mitsue Vieira Cruz Kumasaka

Graduação em Administração. Mestrado em Administração.

E-mail: juliamitsue@hotmail.com

\section{Resumo}

As pressões sociais, governamentais e de fornecedores têm provocado uma série de mudanças na forma como as empresas têm encarado as questões ambientais. $\mathrm{O}$ aumento das exigências impostas pelas legislações, bem como o interesse das empresas em se apresentarem como sustentáveis têm contribuído para a instalação de um ambiente que demanda um conjunto de ações práticas relacionadas ao meio ambiente e à sustentabilidade empresarial. Nesse contexto, o objetivo deste estudo é avaliar o nível de consciência ambiental dos colaboradores da empresa objeto de estudo, tendo em vista o apoio ao planejamento de ações voltadas ao meio ambiente e à sustentabilidade, que a empresa pretende implementar a partir de 2019. A partir de um questionário respondido por 64 dos quase 200 funcionários da empresa, foi aplicado o modelo VAPERCOM, a partir do qual foi possível identificar que, embora os funcionários tenham apresentado níveis que indicam a existência de consciência ambiental, eles não possuem a intenção de consumo ambiental. A partir dos resultados encontrados, conclusões, limitações e sugestões de pesquisas futuras são apresentadas ao final deste estudo.

Palavras-chave: Consciência ambiental. Sustentabilidade. VAPERCOM. Fornecimento de Software.

\begin{abstract}
Social, government and supplier pressures have caused a series of changes in the way companies have viewed environmental issues. The increase in the requirements imposed by legislation, as well as the interest of companies in presenting themselves as sustainable have contributed to the installation of an environment that demands a set of practical actions related to the environment and corporate sustainability. In this context, the objective of this study is to assess the level of environmental awareness of the employees of the company under study, with a view to supporting the planning of actions aimed at the environment and sustainability, which the company intends to implement from 2019 onwards. From a questionnaire answered by 64 of the company's almost 200 employees, the VAPERCOM model was applied, from which it was possible to identify that, although the employees had levels that indicate the existence of environmental awareness, they do not have the intention to consume environmental. Based on the results found, conclusions, limitations and suggestions for future research are presented at the end of this study.
\end{abstract}

Keywords: Environmental awareness. Sustainability. VAPERCOM. Software Supply. 


\section{INTRODUÇÃO}

A pressão do ambiente externo, a partir das exigências dos consumidores, somadas a uma série de regulamentações do Estado, exigem que as empresas se adequem a padrões de produção e de fornecimento de produtos e serviços que visem a preservação ambiental (AGERON; GUNASEKARAN; SPALANZANI, 2012; SANTOS; CARNEIRO; RAMALHO, 2015; SEVERO; DE GUIMARÃES, 2017; VĂTĂMĂNESCU; GAZZOLA; DINCĂ; PEZZETTI, 2017).

Nota-se que há uma grade quantidade de proprietários e gestores de empresas ativamente envolvidos em questões ambientais, pois acreditam nos efeitos positivos que as suas ações podem ter em relação ao meio ambiente, além de benefícios como a conquista de novos clientes, redução de custos, retenção de pessoal e melhoria da imagem da empresa (REVELL, STOKES; CHEN, 2010).

Para que haja o completo alinhamento entre as decisões estratégicas e as atividades operacionais das organizações, Gabler et al. (2017) sugerem que a organização deve abraçar uma filosofia de sustentabilidade empresarial, e desenvolver estratégias que se alinhem a essa filosofia. A partir daí as estratégias devem ser operacionalizadas, executadas e, principalmente, medidas.

Embora, ao longo dos anos, o nível de consciência ambiental da população tenha aumentado (TSENG; HUNG, 2013), o mercado de produtos ambientalmente sustentáveis, ou verdes, não tem acompanhado essa evolução. Esse fenômeno faz com que as expectativas dos indivíduos não sejam atendidas, tornando-os mais céticos em relação às ações realizadas por empresas ambientalmente sustentáveis, incluindo as soluções de Tecnologia da Informação (TI) verde ofertadas nos últimos anos.

$\mathrm{Na}$ busca pelo entendimento de como os funcionários de uma empresa de TI enxergam as questões ambientais, o objetivo principal desta pesquisa é avaliar o nível de consciência ambiental dos colaboradores da empresa objeto de estudo, tendo em vista o apoio ao planejamento de ações voltadas ao meio ambiente e à sustentabilidade, que a empresa pretende implementar a partir de 2019.

O estudo se justifica pela necessidade de se promover a conscientização ambiental entre colabores e clientes das empresas da área de $\mathrm{TI}$ que, embora utilizem o conhecimento como matéria-prima para o desenvolvimento de produtos e serviços, também são consumidores de equipamentos e recursos energéticos que permitem a realização de seu trabalho.

Devido às pressões sofridas por parte dos clientes, parceiros e da comunidade, de maneira geral, a empresa decidiu iniciar um processo de implantação de sua política ambiental, sendo este o passo inicial para o desenvolvimento de uma série de ações voltadas ao meio ambiente e à sustentabilidade empresarial.

A fim de determinar os principais pontos que merecem atenção ao dar início ao desenvolvimento dessas iniciativas, foi aplicado um questionário, adaptado do modelo VAPERCOM, para os colaboradores da empresa, dos quais foram respondidos 64, possibilitando compreender melhor 0 posicionamento dos funcionários em relação ao meio ambiente e às práticas de consumo sustentável.

Desta forma, a partir do conhecimento da percepção ambiental e do perfil de consumo ambiental dos funcionários, poderá ser construída toda a base para as ações da política ambiental da empresa, de modo que as ações possam ser direcionadas e tenham maior nível de efetividade na promoção da consciência 


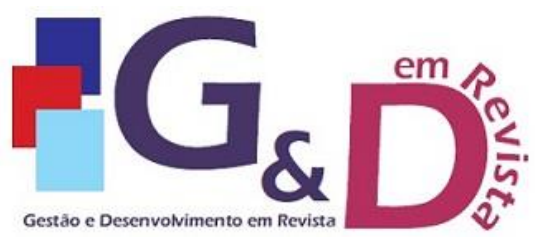

ambiental e da sustentabilidade do negócio.
Gestão e Desenvolvimento em Revista V. 6, N. 2, jul-dez/2020, p. 54-67.

ISSN online: $2446-8738$

Artigo recebido em: $12 / 08 / 2020$

Artigo aprovado em: 11/11/2020

\section{REVISÃO DE LITERATURA}

A fim de melhor compreender os conceitos relacionados à consciência ambiental dos colaboradores e do setor de fornecimento de software, esses temas serão abordados de maneira mais aprofundada nas subseções a seguir.

\subsection{SUSTENTABILIDADE}

O desenvolvimento sustentável no nível macro, ou seja, em Estados, em sociedades e, consequentemente no mundo, depende do comprometimento que as organizações tiverem com a causa (MACKE; GENARI, 2019), já que a sociedade e as corporações são interdependentes (GONÇALVES-DIAS, 2013). Assim, é evidente o papel das corporações na sustentabilidade (GLAVAS, 2014), principalmente considerando que elas representam os recursos produtivos da economia (HAHN ET AL., 2014).

Esse cenário apresenta-se como favorável para que as empresas passem a incrementar os investimentos em melhores práticas, revendo seus processos produtivos (SEVERO; DE GUIMARÃES, 2017), visando a redução das emissões de gases nocivos, redução da eliminação de efluentes e resíduos, economia de energia, proteção das águas e da biodiversidade (SCHWACH et al., 2017).

Apesar do surgimento de muitas boas iniciativas na comunidade empresarial, muitas delas ainda não são divulgadas (MACHADO JUNIOR et al., 2017), ou, quando são, não há padronização dos critérios por parte das empresas. Considerando as empresas do setor de serviços, no qual a empresa objeto deste estudo está inserida, o percentual de organizações que promovem essas ações e as divulgaram para a população é bastante inferior a setores como a indústria, por exemplo (SCHWACH et al., 2017).

A questão é que, ainda que pouco divulgadas, as ações tem sido gradativamente realizadas, e os gestores das organizações têm ampliado um movimento em busca da solução de uma série de problemas ambientais (SEVERO; DE GUIMARÃES, 2017). De acordo com os autores, as preocupações ambientais dos empresários estão relacionadas às forças exercidas pela população, governos, sociedade e mercados.

Essas pressões estão relacionadas principalmente à utilização racional dos recursos naturais, disposição adequada de resíduos e consequente redução de impactos ao meio ambiente. Todas as ações empregadas neste cenário devem ser apoiadas por fortes estratégias de interação e de marketing, fortalecendo as relações com a comunidade, fornecedores e parceiros (SEVERO; DE GUIMARÃES, 2017; VĂTĂMĂNESCU et al., 2017).

Para que uma vantagem competitiva seja alcançada por meio de práticas sustentáveis, é necessário que a empresa seja capaz de integrar, construir e reconfigurar recursos internos e externos para conseguir uma inovação sustentável. Dessa forma, precisa existir um bom desempenho dos funcionários, do marketing e do financeiro (VARADARAJAN, 2017). Para que os colaboradores entendam a importância da sustentabilidade nos processos, produtos e serviços da organização, 
melhorando o seu desempenho e motivação, é preciso que a sua consciência ambiental seja buscada.

\subsection{CONSCIÊNCIA AMBIENTAL DOS COLABORADORES}

Ao longo dos anos, uma série de estudos têm sido realizada a fim de identificar os principais fatores que influenciam no nível de consciência ambiental dos indivíduos. Dentre os principais achados, fatores como a cultura, estruturas ambientais, além dos serviços fornecidos à população em diversos países, tem desempenhado papel relevante no comportamento da população (VICENTEMOLINA; FERNÁNDEZ-SÁINZ; IZAGIRRE-OLAIZOLA, 2013).

Nessa perspectiva, a cultura organizacional afeta todos os tipos de atores e explica valores, crenças ou práticas das organizações que as diferenciam entre si (GIORGI, 2015). A cultura corporativa pode ser dividida em três níveis que interagem de forma dinâmica. O primeiro abrange os chamados artefatos, ou seja, são as estruturas e processos visíveis para os diferentes atores, como vestimenta, linguagem e slogan. O segundo são os valores, o que é considerado certo ou errado entre os colaboradores e gestores como meios para se alcançar um fim, sendo consenso social. O terceiro e último nível são as suposições básicas, quando as crenças entre as pessoas já são tão internalizadas que se tornam inconscientes, sendo construídas ao longo do tempo (SCHEIN, 1993).

A sustentabilidade corporativa não pode ser apenas ações isoladas ou complementares, ela precisa estar presente nos valores organizacionais (SCHONBORN et al., 2018). Dessa forma, não pode estar apenas nos artefatos, como leis que devem ser seguidas ou apenas mais alguns processos estabelecidos pela alta administração, ela precisa estar presente nos valores e quando mais madura, nas suposições básicas dos atores, para que faça sentido e seja realmente internalizada pelos stakeholders.

Com essa abordagem mais profunda, as empresas conseguem adquirir legitimidade com a criação de valor do "fazendo o bem" (SCHÖNBORN et al., 2019). Para isso, é necessário que exista um sentimento de justiça entre os diferentes stakeholders (MACKE; GENARI, 2019). Assim, além de influenciar internamente os seus colaboradores, a organização passa a estimular externamente, como os seus fornecedores (BONN; FISHER, 2011), por exemplo, ao buscar insumos sustentáveis. A gestão com os fornecedores é crucial para a gestão ambiental e quando uma empresa adota as práticas sustentáveis isso impacta em toda a sua cadeia de suprimentos (JABBOURA ET AL., 2014).

Outro ponto importante é o papel do setor de gestão de pessoas para o desenvolvimento e disseminação entre os colaboradores dessa estratégia e dessa cultura voltada para a sustentabilidade. Nas organizações tradicionais ou adeptas do paradigma dominante, o setor de recursos humanos é focado em metas financeiras e precisa passar a objetivar as não-financeiras, pois isso repassa nos valores buscados para os colaboradores, nos treinamentos dados e recompensas oferecidas, além de todas as outras funções da área (MACKE; GENARI, 2019). Pois é a área de gestão de pessoas que possui a capacidade de atrair e reter uma melhor força de trabalho (VARADARAJAN, 2017).

Assim, suas ações também conseguem fomentar o clima organizacional propício para os valores de sustentabilidade serem implementados com sucesso e 
apoio dos atores. Algumas práticas importantes para isso são a promoção de: aprendizagem organizacional, participação no processo decisório, equilíbrio entre a vida profissional e pessoal, além de táticas de longo prazo (MACKE; GENARI, 2019).

É possível que a educação seja o que tem o maior nível de influência sobre as atitudes pró-ambientais dos indivíduos, sendo o conhecimento ambiental adquirido 0 principal influenciador dessas atitudes (VICENTE-MOLINA; FERNÁNDEZ-SÁINZ; IZAGIRRE-OLAIZOLA, 2013; YUAN \& ZUO, 2013). Esse conhecimento pode ser definidos como sendo a capacidade que os indivíduos possuem de identificar símbolos, conceitos ou padrões de comportamento que possuam relação com a proteção ambiental (LAROCHE; BERGERON; BARBAROFORLEO, 2001).

Uma das formas de se promover esta educação, é por meio do fornecimento de feedbacks constantes aos consumidores, de modo que eles possam acreditar que, de fato, estão fazendo a diferença para o planeta (LAROCHE; BERGERON; BARBARO-FORLEO, 2001). Por essa razão, além de adotar políticas sustentáveis e ambientalmente corretas, as empresas devem, por meio de suas equipes de marketing, promover a divulgação dessas ações para o público interno e externo.

Além disso, de acordo com estudo realizado por Tseng \& Hung (2013), é necessário que os aspectos relacionadas às expectativas da população sejam abordados de maneira mais aprofundada. Para os autores, embora o número de pesquisas sobre o tema tenha evoluído, na prática as pessoas ainda não encontram argumentos concretos que atendam às suas expectativas em relação ao consumo ambiental.

O conjunto de estratégias elaborados pelas empresas para divulgação dos seus indicadores ambientais e de sustentabilidade tendem a ter maior efeito sobre consumidores que possuem um alto nível de consciência ambiental. Nesses casos, embora o preço dos produtos ainda se apresente como uma barreira a ser ultrapassada, as pessoas com maior nível de preocupação com o meio ambiente tendem a adaptar o seu comportamento de compra (ZHAO et al., 2014). Esse efeito pode ser potencializado por meio de estratégias de marketing apropriadas (LAROCHE; BERGERON; BARBARO-FORLEO, 2001; ZHAO et al., 2014) adotas pelas empresas, independentemente do segmento de atuação.

\subsection{O MERCADO DE SOFTWARE}

O mercado de Tecnologia da Informação (TI) no Brasil tem apresentado bons resultados ao longo dos últimos anos. De acordo com dados da Brasscom (2018), em 2017 os setores de TI, comunicação e telecomunicações movimentaram, no Brasil, por volta de $\mathrm{R} \$ 468$ bilhões (cerca de $7 \%$ do PIB do país). Ainda de acordo com os relatórios apresentados por essa instituição, o Brasil é o maior mercado de TI da América Latina, apresentando $49,3 \%$ da receita proveniente desse setor na região. As projeções para o mercado são de crescimento, com uma previsão de $10,5 \%$ ao ano na América Latina e 9,5\% no Brasil até 2022 (BRASSCOM, 2018).

Muito do crescimento do setor se deve ao fato de que a digitalização tem aumentado ao longo dos anos, não apenas no âmbito econômico, mas também no âmbito social (ZACKIEWICZ, 2014). Para o autor, fatores como difusão de softwares open source, além de incentivos para a inovação e de um arcabouço legal e 
normativo fizeram com que o mercado de softwares tenha se fortalecido.

Sob uma perspectiva menos otimista, Cerqueira, Salvador, \& Brazil (2013) apontam que, apesar das evoluções ocorridas no mercado brasileiro, a indústria de softwares ainda precisa percorrer um longo caminho vislumbrando alcançar países como os EUA e Japão.

Assim como ocorre com os indicadores ambientais, é necessário que as práticas voltadas à melhoria de processos e à qualidade de produtos sejam divulgadas (LUZ, K. S; LOPES, R. F. P.; PAULO DA SILVA, 2016). Esse conjunto de atitudes torna-se ainda mais relevante quando se analisa o processo de internacionalização das empresas brasileiras, cada vez mais comum dentre aquelas que encontram oportunidades a partir de suas redes de relacionamentos (CORTEZIA; DE SOUZA, 2011).

\section{PROCEDIMENTOS METODOLÓGICOS}

O presente estudo foi desenvolvido sobre a perspectiva quantitativa e descritiva, visando mensurar a percepção do consumidor quanto a sua consciência de consumo sustentável. Considerando como propósito apresentar o comportamento de consumo dos colaboradores da organização (MALHOTRA, 2012). Sendo composta pela amostragem não probabilística por conveniência, por se tratar da modalidade de amostragem em que o pesquisador seleciona os elementos a que tem acesso (LEMESHOW; LEVY, 1980).

Como método, aplicou-se o modelo Variável Ambiental; Percepção e Comportamento do Consumidor - VAPERCOM, desenvolvido pela autora Loreni Brandalise (2008), que busca avaliar a percepção do consumidor considerando a variável ambiental nas etapas da ACV (Análise do Ciclo de Vida do produto).

Esse considera três elementos que atuam sobre o consumidor no macro ambiente: a variável ambiental, os estímulos (internos e externos) e as influências (sociais, de marketing e situacionais) que incidem sobre outros três elementos associados ao produto e ao consumidor: a ACV, a percepção e o processo de compra (BRANDALISE, 2008). Essa estrutura do modelo está representada na Figura 1.

Figura 1 - Modelo conceitual VAPERCOM

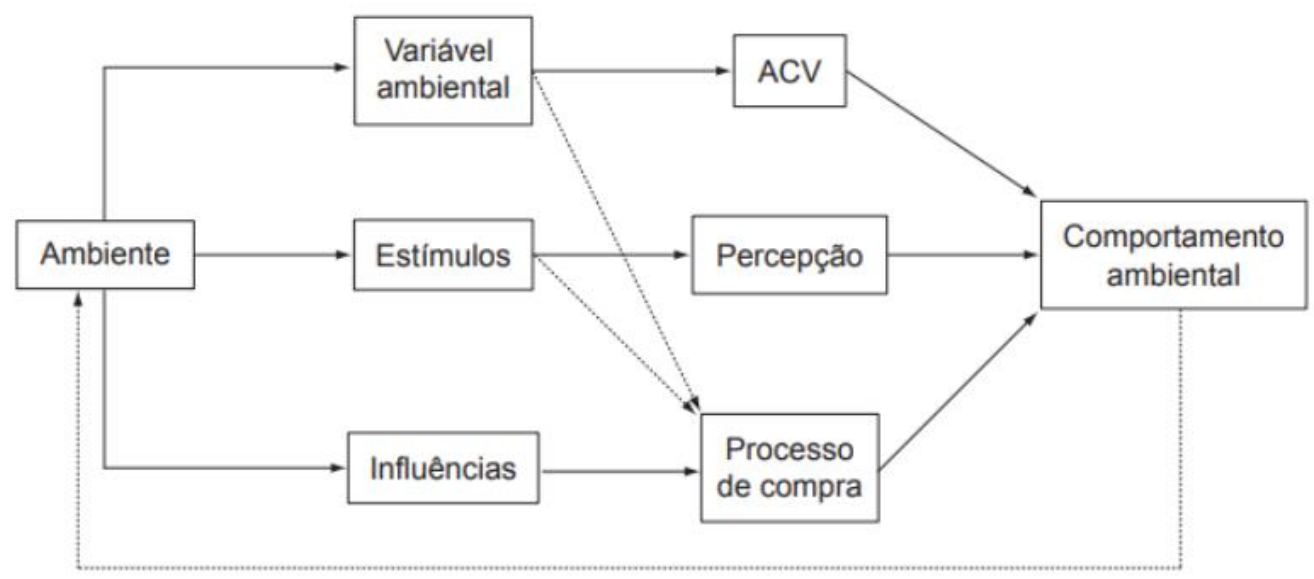

Fonte: Brandalise (2008) 
A aplicação do VAPERCOM, a qual é explorada ao decorrer do presente trabalho, é constituído em quatro fases: (1) caracterização do produto e do potencial consumidor; (2) identificação do perfil do pesquisado, da percepção ambiental, do consumo ecológico e das etapas da ACV; (3) identificação das discrepâncias (gaps) entre as características ambientais do produto e aquelas que o consumidor percebe; e, por fim, (4) definição de oportunidades de ações (de incremento e/ou ajustes).

Validado em diversas pesquisas (BRANDALISE, BERTOLINI, ROJO, LEZANA, \& POSSAMAI, 2009; FUJIHARA, BRANDALISE, BERTOLINI, \& ROJO, 2017; SILVA, SANDRI, ROVARIS, \& BRANDALISE, 2019), o modelo foi adaptado para este estudo considerando que, neste caso, o objetivo deste trabalho é identificar o nível de percepção ambiental e potenciais características de consumo ecológico entre os funcionários da empresa objeto de estudo.

A população dos colaboradores da organização estudada compreende todos os 200 profissionais registrados e ativos durante o periodo da aplicação desta pesquisa. A coleta dos dados foi realizada, entre os períodos dos meses de novembro e dezembro do ano de 2018, onde, foram coletados 64 questionários (que pode ser consultado no apêndice no docoumento suplementar), por meio de abordagem, entrega e recolhimento dos formulários aos profissionais de forma presencial pelos pesquisadores.

As próximas duas sub-seções se referem, respectivamente, a caracterização da empresa estudada e o modelo de mensuração dos dados.

\subsection{CARACTERIZAÇÃO DA EMPRESA}

A empresa objeto do estudo atua no ramo de desenvolvimento de softwares e prestação de serviços de consultoria. Há mais de 10 anos no mercado, possui hoje aproximadamente 200 colaboradores cuja área de formação abrange o setor de tecnologia, incluindo Ciência da Computação, Informática, Sistemas de Informação, Tecnologia em Análise e Desenvolvimento de Sistemas, além de áreas como o Direito, Administração, Marketing e Contabilidade.

Devido às pressões sofridas por parte dos clientes, parceiros e da comunidade, de maneira geral, a empresa decidiu iniciar um processo de implantação de sua política ambiental, sendo este o passo inicial para o desenvolvimento de uma série de ações voltadas ao meio ambiente e à sustentabilidade empresarial.

A fim de determinar os principais pontos que merecem atenção ao dar início ao desenvolvimento dessas iniciativas, foi aplicado um questionário, adaptado do modelo VAPERCOM, para os colaboradores da empresa, dos quais foram respondidos 64, possibilitando compreender melhor o posicionamento dos funcionários em relação ao meio ambiente e às práticas de consumo sustentável.

\subsection{MODELO DE MENSURAÇÃO DOS DADOS}

Os dados referentes à percepção ambiental foram tabulados de acordo com as diretrizes do modelo VAPERCOM, conforme evidenciado na Tabela 1. Na tabela são alocados os pesos e realizada a elaboração do grau de percepção.

A pesquisa foi aplicada por meio de um questionário divido em três quadros, sendo o primeiro (Conjunto 1) referente à caracterização dos entrevistados, o 
segundo (Conjunto 2) relacionado à percepção ambiental e o terceiro (Conjunto 3) abordando o perfil de consumo ecológico. Os três conjuntos antes citados se encontram no Apêndice deste artigo.

As questões foram tabuladas multiplicando-se o número de ocorrências de cada resposta (a) pela respectiva pontuação a ela atribuída (b). Somam-se todos os resultados (c) e divide-se o mesmo pelo número de questões respondidas que se relacionam à percepção ecológica $(\mathrm{d})$.

Tabela 1 - Alocação de pesos e elaboração do grau de percepção

\begin{tabular}{ccc}
\hline (a) № Respostas & (b) Valores & (a X b) Resultado \\
\hline A & 4 \\
B & 3 \\
C & 2 \\
D & 1 \\
E & 0 \\
\hline
\end{tabular}

(c) Soma dos resultados

(d) № de questões

$(\mathrm{e}=\mathrm{c} / \mathrm{d})$ Resultado

Fonte: Brandalise (2008)

Nota. Os valores atribuídos às questões alternativas de respostas para a classificação foram: para $A$ $($ sempre $)=4$ pontos; para $B$ (frequentemente) $=3$ pontos; para $C$ (algumas vezes) $=2$ pontos; para $\mathrm{D}$ (pouquíssimas vezes) $=1$ ponto; e para $E$ (nunca) $=0$.

Com o intuito de viabilizar os cálculos de mensuração dos indicadores do conjunto da percepção ambiental, utiliza-se a classificação apresentada na Tabela 2. As escalas de classificação obedecem a intervalos de 0,7 pontos e são classificadas alfabeticamente a fim de facilitar a visualização dos resultados, onde: A) a partir de intervalos entre 3,3 e 4,0; B) entre 2,5 e 3,2; C) entre 1,7 e 2,4; D) entre 0,9 e 1,6; e E) até 0,8 .

Tabela 02 - Classificação do grau de percepção ambiental Grau de consumo de produtos ecologicamente corretos
A) Possui alta percepção ecológica
B) Possui percepção ecológica
C) Possui potenciais traços de percepção ambiental
D) Possui poucos traços de percepção ambiental
E) Não possui percepção ambiental
Fonte: Adaptado de Brandalise (2008) Valores Até 0,8

A partir dos dados da seção três do questionário, os dados foram tabulados utilizando-se novamente a estrutura da Tabela 1. A seguir, foi realizada a classificação conforme modelo apresentado na Tabela 3, obedecendo a mesma pontuação atribuída aos itens da seção dois do questionário. 
Tabela 03 - Classificação do comportamento de compra ecológica Grau de consumo de produtos ecologicamente corretos
A) Consumidor ecológico
B) Grande possibilidade de tornar-se um consumidor ecológico
C) Potencial possibilidade de tornar-se um consumidor ecológico
D) Fraca possibilidade de tornar-se um consumidor ecológico
E) Não é um consumidor ecológico Valores

Fonte: Adaptado de Brandalise (2008)

Entre 3,3 e 4,0

Entre 2,5 e 3,2

Entre 1,7 e 2,4

Entre 0,9 e 1,6 Até 0,8

Os resultados obtidos a partir da aplicação do modelo são apresentados na seção de resultados e discussões a seguir.

\section{RESULTADOS E DISCUSSÕES}

A partir das respostas obtidas, uma série de análises pode ser realizada, com o intuito de traçar o perfil ambiental dos colaboradores da empresa. Do total de 64 respondentes, $52(81,25 \%)$ foram homens e $12(18,75 \%)$ mulheres com idade predominante entre 21 e 30 anos (76,56\%). Além disso, entre os respondentes não foram identificados indivíduos sem formação superior, sendo que $32,81 \%$ possuem também cursos de especialização.

A partir da aplicação do modelo sobre os dados coletados na pesquisa, foi possível identificar que os pesquisados possuem percepção ecológica (Tabela 4). Isso pode estar relacionado ao fato de que, embora as iniciativas ainda precisem avançar intensamente no Brasil (SCHWACH et al., 2017), as empresas têm demonstrado cada vez mais interesse em incorporar em suas práticas os conceitos de sustentabilidade (PORTER; KRAMER, 2006).

Tabela 4 - Alocação de pesos e elaboração do nível percepção ecológica

\begin{tabular}{lccc}
\hline Respostas & (C) No Respostas & (B) Valores & (A * B) Resultado \\
\hline Sempre & 178 & 4 & 712 \\
Frequentemente & 147 & 3 & 441 \\
Algumas Vezes & 62 & 2 & 124 \\
Pouquíssimas Vezes & 40 & 1 & 40 \\
Nunca & 21 & 0 & 0 \\
\hline (C) Soma dos resultados & & & 1317 \\
(D) Questões respondidas & & & 448 \\
(E) Resultado & & & 2,94 \\
\hline
\end{tabular}

Nota. O índice de 2,94 aponta a existência de percepção ecológica entre os entrevistados, conforme detalhado na Tabela 2. Fonte: Dados da pesquisa (2017)

A partir de uma série de campanhas e informações cada dia mais presentes nas mídias, apontadas por $90,6 \%$ dos entrevistas como sendo a principal forma de obtenção de informações sobre questões ambientais no dia-a-dia, pode-se perceber uma forte tendência à mudanças comportamentais dos indivíduos, o que deve ser incentivado por meio de uma constante educação ambiental (ZSÓKA et al., 2013), seja ela no ambiente acadêmico ou profissional.

De maneira geral, os funcionários da empresa apresentaram características de percepção ecológica, entretanto, o nível de percepção apresentado pelas mulheres $(3,14)$ foi superior àquele apresentado pelos homens $(2,84)$. 
Em relação à idade, foi possível identificar que o índice de consciência ambiental é mais forte entre os indivíduos mais jovens, com idade até 20 anos $(3,05)$ do que aqueles com idade a partir dos 21 anos (2,9), o que se justifica por uma série de políticas que têm sido adotadas no sentido de educar as pessoas para o desenvolvimento sustentável e para mudanças no estilo de vida relacionadas ao consumo (HIBBERT et al., 2013; YUAN \& ZUO, 2013).

A partir do momento em que a empresa passa a explorar essas lacunas, investindo na educação ambiental dos colaboradores e divulgando os resultados obtidos por meio de estratégias eficientes de marketing, aos seus clientes, fornecedores, parceiros e à comunidade, subsidiada pela política ambiental adotada, este passa a ser um grande diferencial competitivo para a organização (PORTER \& LINDE, 1995).

Apesar do resultado sobre a percepção ambiental, a perspectiva da intenção de consumo ambiental apresentou índices inferiores ao antes apresentado. Desta forma, embora haja percepção ecológica entre os membros da equipe, a análise do consumo ecológico apresentou valores abaixo (Tabela 5) da expectativa para indivíduos que demonstram características de consciência ambiental.

Tabela 5 - Alocação de pesos e elaboração do nível de consumo ecológico

\begin{tabular}{lccc}
\hline Respostas & (C) $N$ No Respostas & (B) Valores & (A * B) Resultado \\
\hline Sempre & 27 & 4 & 108 \\
Frequentemente & 89 & 3 & 267 \\
Algumas Vezes & 205 & 2 & 410 \\
Pouquíssimas Vezes & 218 & 1 & 218 \\
Nunca & 165 & 0 & 0 \\
\hline (C) Soma dos resultados & & & 1003 \\
(D) Questões respondidas & & & 704 \\
(E) Resultado & & & 1,42 \\
\hline
\end{tabular}

Fonte: Dados da pesquisa (2017).

Nota. $\mathrm{O}$ índice de 1,42 indica a fraca possibilidade de esses indivíduos se tornarem consumidores ecológicos, conforme detalhado na tabela 3.

De acordo com os dados desta pesquisa, $18,8 \%$ dos pesquisados nunca levam em consideração as variáveis ambientais no momento de adquirir um produto, enquanto $37,5 \%$ têm essa percepção pouquíssimas vezes. $17,2 \%$ não se deixam influenciar por propagandas em relação aos produtos ambientalmente corretos, e um número de $40,6 \%$ permite pouquíssimas vezes que isso aconteça.

Esse fenômeno, em parte, pode ser justificado pelo fato de que muitos produtos verdes disponíveis no mercado não atendem às expectativas da população (TSENG; HUNG, 2013). São as lacunas existentes entre aquilo que é pregado pelas organizações e aquilo que, de fato, é entregue ao consumidor, que fazem com que, embora a população apresente características que remetem à percepção ambiental, acabe não adquirindo os produtos ambientalmente corretos e sustentáveis.

Por essa razão, é necessário que as empresas não apenas adotem uma filosofia voltada à sustentabilidade e ao meio ambiente, mas também busquem formas de operacionalizá-la, divulgando seus resultados obtidos com a prática, avaliando e medindo o desempenho dessas estratégias (GABLER et al., 2017). Esta filosofia deve incluir ações como a elaboração de uma política ambiental sólida, e que seja de conhecimento de todos os colaboradores, clientes, parceiros e fornecedores da organização. 


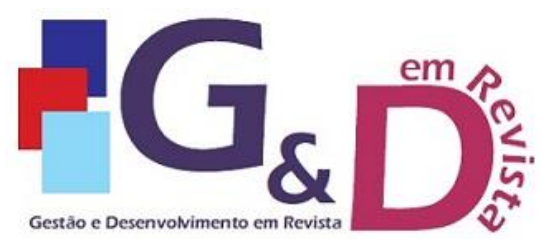

Gestão e Desenvolvimento em Revista V. 6, N. 2, jul-dez/2020, p. 54-67.

ISSN online: $2446-8738$

Artigo recebido em: $12 / 08 / 2020$

Artigo aprovado em: 11/11/2020

\section{CONCLUSÕES}

Foi proposto pelo objetivo deste trabalho a identificação do nível de percepção ecológica e de intenção de consumo ambiental dos funcionários de uma empresa fornecedora de softwares e serviços de consultoria. Foi possível, por meio da aplicação e análise dos indicadores fornecidos pelo método VAPERCOM (BRANDALISE, 2008), verificar o perfil do pensamento e do consumo ecológico dos colaboradores da organização em estudo.

Apesar de estar limitado a uma única empresa, o estudo apresentou contribuições teóricas e práticas, validando proposições de estudos anteriores, e identificando uma série de similaridades entre o público abordado nesta pesquisa e aqueles abordados em outros estudos realizados anteriormente acerca da temática. Além disso, apresentou uma proposta para analisar a percepção e o consumo ecológico dos colaboradores de organizações que buscam entender a percepção do pensamento e consumo ecológico de seus empregados para assim alinhar e/ou desenvolver estratégias na área ambiental das próprias instituições.

Cabe às organizações, incluindo nesse contexto a empresa objeto deste estudo, utilizar essa necessidade de mudança a seu favor, de modo que a adoção de políticas sustentáveis se torne presente nas estratégias dessas instituições. Nesse sentido, o presente estudo pode colaborar como um primeiro passo para identificar o comportamento dos funcionários dessas empresas.

Como limitação, aponta-se que o número de respondentes dessa pesquisa não é generalizável ao comportamento total da organização, servindo os dados coletados como um direcionador para que a organização possa direcionar suas estratégias ambientais ao perfil ecológico de seus colaboradores.

Como sugestões de trabalhos futuros, outras pesquisas podem ser realizadas a fim de avaliar quantitativamente o impacto da percepção ambiental nas atitudes de consumo ambiental nas empresas de TI. Além disso, estudos comparativos entre as percepções de funcionários e clientes das empresas poderiam contribuir na definição das estratégias ambientais a serem adotadas por essas empresas, garantindo maior nível de efetividade das ações implementadas.

\section{REFERÊNCIAS}

AGERON, B.; GUNASEKARAN, A.; SPALANZANI, A. Sustainable supply management: an empirical study. International Journal of Production Economics, v. 140 , n. 1, p. 168-182, 2012.

BONN, I.; FISHER, J. Sustainability: the missing ingredient in strategy. Journal Of Business Strategy, v. 32, n. 1, p.5-14, 2011.

BRANDALISE, L. T. Modelo de suporte à gestão organizacional com base no comportamento do consumidor considerando sua percepção da variável ambiental nas etapas da análise do ciclo de vida do produto. Tese (Doutorado em Engenharia de Produção) - Universidade Federal de Santa Catarina, 2008.

BRANDALISE, L. T. L. T., BERTOLINI, G. R. F., ROJO, C. A., LEZANA, Á. G. R., \& POSSAMAI, O. A percepção e o comportamento ambiental dos universitários em relação ao grau de educação ambiental. Revista Gestão \& Produção, v. 16, n. 2, p. 


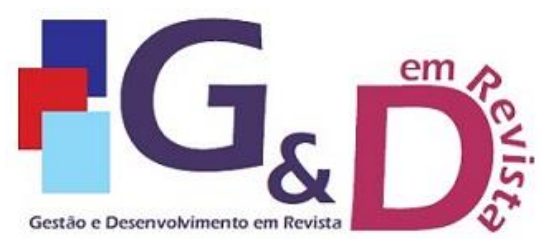

Gestão e Desenvolvimento em Revista V. 6, N. 2, jul-dez/2020, p. 54-67.

ISSN online: $2446-8738$

Artigo recebido em: $12 / 08 / 2020$

Artigo aprovado em: 11/11/2020

273-285, 2009.

BRASSCOM - INTELIGêNCIA E INFORMAÇÃO. Relatório Setorial: mercado de tic. São Paulo: Brasscom, 2018. 50 p. Disponível em: https://docs.google.com/viewerng/viewer?url=https://brasscom.org.br/wpcontent/uploads/2020/04/BRI2-2018-003-Relat\%C3\%B3rio-Setorialv71.pdf\&hl=pt_BR. Acesso em: 25 out. 2019.

CERQUEIRA, L. S.; SALVADOR, U.; BRAZIL, U. Reflexões sobre a intervenção estatal para o desenvolvimento da indústria brasileira de software. UNIFACS, v. 14, n. 2, p. 232-258, 2013.

CORTEZIA, S. L. D.; DE SOUZA, Y. S. An analysis of the internationalization of small Brazilian software companies. Brazilian Business Review (English Edition), v. 8, n. 51, p. 23-43, 2011.

DA SILVA, M. L., SANDRI, E. C., ROVARIS, N. R. S., BRANDALISE, L. T. Percepção ambiental dos clientes de um restaurante universitário, sobre a análise do ciclo de vida (acv) das refeições. Revista Metropolitana de Sustentabilidade, v. 9, n. 2, p. 37-52, 2019.

FUJIHARA, H. M. L.; BRANDALISE, L. T.; BERTOLINI, G. R. F.; ROJO, C. A. Análise da percepção ambiental dos microempreendedores da cidade de catanduvas-PR. Revista Eletrônica Científica do CRA-PR-RECC, n. 4, n.1, p. 6478. 2017.

GABLER, C. B., PANAGOPOULOS, N., VLACHOS, P. A., \& RAPP, A. Developing an Environmentally Sustainable Business Plan: An International B2B Case Study. Corporate Social Responsibility and Environmental Management, v. 24, n. 4, p. 261-272, 2017.

GIORGI, S.; LOCKWOOD, C.; GLYNN, M. A. The Many Faces of Culture: Making Sense of 30 Years of Research on Culture in Organization Studies. The Academy of Management Annals, v. 9, n. 1, p. 1-54, 2015.

GLAVAS, A.; MISH, J. Resources and Capabilities of Triple Bottom Line Firms: Going Over Old or Breaking New Ground?. Journal Of Business Ethics, v. 127, n. 3, p.623-642, 2014.

GONCALVES-DIAS, S.L.F.; HERRERA, C.B.; CRUZ, M.T.S.. Desafios (e dilemas) para inserir "Sustentabilidade" nos currículos de administração: um estudo de caso. RAM, Rev. Adm. Mackenzie [online]., v. 14, n.3, p.119-153, 2013.

HAHN, PINKSE, J., PREUSS, L., \& FIGGE, F. Tensions in Corporate Sustainability: Towards an Integrative Framework. Journal Of Business Ethics, v. 127, n. 2, p.297-316, 2014.

HIBBERT, J. F. DICKINSON, J. E., GÖSSLING, S., \& CURTIN, S. Identity and tourism mobility: An exploration of the attitude-behaviour gap. Journal of 
Sustainable Tourism, v. 21, n. 7, p. 999-1016, 2013.

JABBOUR, A. B., JABBOUR, C., GOVINDAN, K., KANNAN, D., \& ARANTES, A. F. Mixed methodology to analyze the relationship between maturity of environmental management and the adoption of green supply chain management in Brazil. Resources, Conservation and Recycling, v. 92, p. 255-267, 2014.

LAROCHE, M.; BERGERON, J.; BARBARO-FORLEO, G. Targeting consumers who are willing to pay more for environmentally friendly products. Journal of Consumer Marketing, v. 18, n. 6, p. 503-520, 2001.

LEMESHOW, S; LEVY, P. S. Sampling for health professionals. Belmont: Lifetime Learning Publications, 1980.

LUZ, K. S; LOPES, R. F. P.; PAULO DA SILVA, P. Mapeamento da utilização de modelos MPS. br e CMMI para melhorias no processo de desenvolvimento de software no mercado nacional. Tecnologias em Projeção, v.7, n.1, p. 62-69, 2016.

MACHADO JUNIOR, C.; RIBEIRO, D. M. N.M.; MAZZALI, L.; BAZANINI, R.; DA SILVA PEREIRA, R. Indicadores de governança e sustentabilidade de empresas brasileiras. Revista de Administração da UNIMEP, v. 15, n. 2, p. 31-55, 2017.

MACKE, J.; GENARI, D. Systematic literature review on sustainable human resource management. Journal Of Cleaner Production, v. 208, p.806-815, 2019.

MALHOTRA, N. K.; DASH, S. Marketing research: An applied orientation. Pearson, 2016.

PORTER, M. E.; KRAMER, M. R. Strategy and society: The link between competitive advantage and corporate social responsibility. Harvard Business Review, v. 84, n. 12, p. 78-92, 2006.

PORTER, M. E.; LINDE, C. VAN DER. Green and competitive: ending the stalemate. Harvard Business Review, v. 28, n. 6, p. 128-129, 1995.

REVELL, A.; STOKES, D.; CHEN, H. Small businesses and the environment: Turning over a new leaf? Business Strategy and the Environment, v. 19, n. 5, p. 273-288, 2010.

SANTOS, J. G.; CARNEIRO, V. C. V.; RAMALHO, Â. M. C. Sustentabilidade e produção mais limpa: um estudo sobre as implicações na vantagem competitiva empresarial. Revista Metropolitana de Sustentabilidade, v. 5, n. 2, p. 34-48, 2015.

SCHEIN, E.H. Defining Organizational Culture. In: SHAFRITZ, J.M.; OTT, J.S.; JANG, Y.S. (Eds). Classics of Organization Theory. London: Thomson, 1993.

SCHÖNBORN, G. BERLIN, C., PINZONE, M., HANISCH, C., GEORGOULIAS, K., \& LANZ, M. Why social sustainability counts: The impact of corporate social sustainability culture on financial success. Sustainable Production and 
Consumption, v. 17, p. 1-10, 2019.

SCHWACH, R. G., JUNIOR, C. M., DA SILVA PEREIRA, R., \& RIBEIRO, D. M. N. M. Divulgação de indicadores de sustentabilidade pelas empresas com governança corporativa. v. 15, n. 3, p. 457-470, 2017.

SEVERO, E.; DE GUIMARÃES, J. Trajetórias e perspectivas da sustentabilidade e práticas ambientais: Uma pesquisa bibliométrica. Revista Metropolitana de Sustentabilidade, v. 7, n. 2, p. 93-114, 2017.

TSENG, S. C.; HUNG, S. W. A framework identifying the gaps between customers' expectations and their perceptions in green products. Journal of Cleaner Production, v. 59, p. 174-184, 2013.

VARADARAJAN, R. Innovating for sustainability: a framework for sustainable innovations and a model of sustainable innovations orientation. Journal of the Academy of Marketing Science, v. 45, n. 1, p. 14-36, 2017.

VĂTĂMĂNESCU, E. M., GAZZOLA, P., DINCĂ, V. M., \& PEZZETTI, R. Mapping entrepreneurs' orientation towards sustainability in interaction versus network marketing practices. Sustainability (Switzerland), v. 9, n. 9, 2017.

VICENTE-MOLINA, M. A.; FERNÁNDEZ-SÁINZ, A.; IZAGIRRE-OLAIZOLA, J. Environmental knowledge and other variables affecting pro-environmental behaviour: Comparison of university students from emerging and advanced countries. Journal of Cleaner Production, v. 61, p. 130-138, 2013.

YUAN, X.; ZUO, J. A critical assessment of the Higher Education for Sustainable Development from students' perspectives - A Chinese study. Journal of Cleaner Production, v. 48, p. 108-115, 2013.

ZACKIEWICZ, M. A economia do software e a digitalização da economia. Revista Brasileira Inovação, v. 14, n. 2, p. 313-336, 2014.

ZHAO, H. H., GAO, Q., WU, Y. P., WANG, Y., \& ZHU, X. D. What affects green consumer behavior in China? A case study from Qingdao. Journal of Cleaner Production, v. 63, p. 143-151, 2014.

ZSÓKA, Á., SZERÉNYI, Z. M., SZÉCHY, A., \& KOCSIS, T. Greening due to environmental education? Environmental knowledge, attitudes, consumer behavior and everyday pro-environmental activities of Hungarian high school and university students. Journal of Cleaner Production, v. 48, p. 128-138, 2013. 